\title{
Identification of a Polyketide Synthase Gene in the Synthesis of Phleichrome of the Phytopathogenic Fungus Cladosporium phlei
}

\author{
Kum-Kang So', Yun-Jo Chung ${ }^{2}$, Jung-Mi Kim³, Beom-Tae Kim', Seung-Moon Park', and Dae-Hyuk Kim ${ }^{1, *}$
}

\begin{abstract}
Phleichrome, a pigment produced by the phytopathogenic fungus Cladosporium phlei, is a fungal perylenequinone whose photodynamic activity has been studied intensively. To determine the biological function of phleichrome and to engineer a strain with enhanced production of phleichrome, we identified the gene responsible for the synthesis of phleichrome. Structural comparison of phleichrome with other fungal perylenequinones suggested that phleichrome is synthesized via polyketide pathway. We recently identified four different polyketide synthase (PKS) genes encompassing three major clades of fungal PKSs that differ with respect to reducing conditions for the polyketide product. Based on in silico analysis of cloned genes, we hypothesized that the non-reducing PKS gene, Cppks1, is involved in phleichrome biosynthesis. Increased accumulation of Cppks1 transcript was observed in response to supplementation with the application of synthetic inducer cyclo-( ${ }_{L}-$ Pro- $_{L}-$ Phe). In addition, heterologous expression of the Cppks1 gene in Cryphonectria parasitica resulted in the production of phleichrome. These results provide convincing evidence that the Cppks1 gene is responsible for the biosynthesis of phleichrome.
\end{abstract}

\section{INTRODUCTION}

Cladosporium phlei (George) de Vries belongs to a group of hypomycetous fungus that causes purple eyespot disease in timothy (Phleum pratense). This disease is one of the most common foliar diseases of timothy, which is easily distinguishable by eye-shaped spots of light greyish-fawn centers with pur-

\footnotetext{
${ }^{1}$ Institute for Molecular Biology and Genetics, Chonbuk National University, Jeonju 561-756, Korea, ${ }^{2}$ Physical Lab., Center for University-wide Research Facilities, Chonbuk National University, Jeonju 561-756, Korea, ${ }^{3}$ Department of Bio-Environmental Chemistry, Institute of Life Science and Natural Resources, Wonkwang University, Iksan 570-749, Korea, ${ }^{4}$ Research Center of Bioactive Materials, Chonbuk National University, Jeonju 561-756, Korea

*Correspondence: dhkim@jbnu.ac.kr
}

Received 22 July, 2015; revised 21 September, 2015; accepted 30 September, 2015; published online 25 November, 2015

Keywords: Cladosporium phlei, Cryphonectria parasitica, heterologous expression, phleichrome, polyketide synthase ple margins on plant leaves. C. phlei produces a characteristic deep red pigment, phleichrome, in mycelia and culture medium (Yoshihara et al., 1975). Phleichrome, a derivative of 4,9dihydroxyperylene-3,10-quinone, is a member of a group of fungal perylenequinones. Perylenequinones that share the same 4,9-dihydroxyperylene-3,10-quinone chromophore exhibit photodynamic activities and act as photosensitizers because they contain a core chromophore of phenolic quinone that can absorb light energy and produce reactive oxygen species (ROS) such as the hydroxyl radical $\left(\mathrm{OH}^{\circ}\right)$, superoxide anion $\left(\mathrm{O}_{2}{ }^{-}\right)$, hydrogen peroxide $\left(\mathrm{H}_{2} \mathrm{O}_{2}\right)$, and singlet oxygen $\left({ }^{1} \mathrm{O}_{2}\right)$ (Daub and Ehrenshaft, 2000; Daub et al., 2005; Liao and Chung, 2008). The fact that many phytopathogenic fungi produce perylenequinone pigments suggests that perylenequinone pigments may be virulence factors because they produce ROS, which can kill host cells, thereby facilitating fungal invasion. Perylenequinone pigments are therefore considered to be lightactivated, non-host-selective phytotoxins (Daub et al., 2005; Liao and Chung, 2008). Recently, perylenequinones have gained attention because of their therapeutic potential as photosensitizers for photodynamic therapy (PDT) (Hudson and Towers, 1991). Among perylenequinones, the photodynamic activity of phleichrome has been studied in detail (Olivo and Chin, 2006), and it has been used as a pharmacophore to produce various derivatives (Du and Maunder, 2010). In our previous studies, we established a culture system (Lee et al., 2007), genetic manipulation protocol (Kim et al., 2009), and inducer supplementation protocol for mass production of phleichrome (So et al., 2015) from C. phlei. However, the gene responsible for the synthesis of phleichrome has yet to be determined.

Thus, our goal in this study was to identify the gene responsible for biosynthesis of phleichrome. We tested whether Cppks1, which encodes a polyketide synthase (PKS), is the PKS gene responsible for phleichrome biosynthesis by analyzing transcription of Cppks1 in response to a synthetic inducer. Furthermore, we expressed Cppks1 in the chestnut blight fungus Cryphonectria parasitica, which does not produce phleichrome but produces bisanthraquinones such as skyrin, oxyskyrin and rugulosin via the polyketide pathway (Shibata, 1973).

\section{MATERIALS AND METHODS}

Fungal strains, culture media, and growth conditions Cladosporium phlei (ATCC 36193) was stored in the form of 
frozen agar plugs containing actively growing young hyphae in $5 \%$ DMSO solution at $-70^{\circ} \mathrm{C}$ and maintained on potato dextrose agar (PDA) as described previously (Lee et al., 2007). Culture conditions and methods for phleichrome induction using 150 $\mu \mathrm{M}$ cyclo-(L-Pro-L-Phe) have been described previously (So et al., 2015). Details of the chemical synthesis of the cyclo-(L-PheL-Pro) inducer are provided in our previous study (So et al., 2015). Mycelia on cellophane membranes overlaying agar plates were collected and lyophilized as described previously, and stored until use (So et al., 2015). All chemicals were obtained from Sigma-Aldrich Co. (USA), unless otherwise specified.

\section{Analysis of Cppks1 transcript expression}

RNA from mycelia grown on cellophane layered on top of PDA media supplemented with $150 \mu \mathrm{M}$ cyclo-(L-Pro-L-Phe) inducer was extracted as described previously (Park et al., 2004). Northern blot analysis was used to compare the level of Cppks1 (GenBank accession no. JX129223) transcript before and after induction. Semi-quantitative analysis of the accumulation of Cppks1 transcript was performed by RT-PCR using total RNA extracted from cultures at 6 and 18 days after inoculation with and without induction, as described previously (Park et al., 2012). Cppks1 transcript levels were compared with those of another PKS gene encoding a highly-reducing (HR)-PKS, namely Cppks3 (GenBank accession no. JX129225), and normalized to levels of the gene encoding beta-tubulin $(\beta$-tub) as an internal control (Choi and Nuss, 1990). Analyses were conducted at least twice, in triplicate for each transcript, from at least two independent RNA preparations of the same sample with primers specific for $\beta$-tub, Cppks3, and Cppks1 genes. Primer pairs for each gene are described in Table 1. To determine relative phleichrome content from the corresponding mycelia, phleichrome was extracted from mycelia using ethyl acetate (EtOAc) and compared using thin-layer chromatography (TLC) on a silica gel with a resolving solution $\left(\mathrm{CH}_{2} \mathrm{Cl}_{2} / \mathrm{MeOH}=\right.$ 19:1, v/v), as described previously (Yi et al., 2011).

\section{Construction of a Cppks1 expression cassette for heterologous expression}

We selected a genomic fosmid clone containing full-length Cppks1 from our previous study. The deduced Cppks1 sequence contained no introns, and the protein product of CpPKS1 consisted of 2,174 codons, with an estimated molecular mass of $235.1 \mathrm{kDa}$ and a pl of 6.02. To construct an expression vector for $C$. parasitica, the ORF of Cppks1 was placed between the strong 188-bp promoter element of the C. parasitica cryparin gene (Crp1) (Kwon et al., 2009) and the terminator of $A$. nidulans trp $C$ gene (Mullaney et al., 1985) from the fungal transforming vector pSilent-Dual1 (Nguyen et al., 2008) using an overlap extension PCR method. The resulting fusion construct was digested with Notl and ligated into Notl-digested recombinant pBluescriptll SK $(+)$ vector carrying the $1.7-\mathrm{kb}$ Xhol fragment of pSilent-Dual 1 encoding the geneticin resistance cassette (Nguyen et al., 2008). The resulting expression vector p188CpPKS1 was sequenced and then used to transform C. parasitica EP155/2 strain (ATCC 38755).

Protoplast preparation and transformation were performed as described previously (Churchill et al., 1990; Kim et al., 1995). Transformants were selected from agar plates supplemented with $150 \mu \mathrm{g} / \mathrm{ml}$ geneticin (Invitrogen, USA), passaged three to four times on selective media, and single-spore isolated, as described previously (Baek et al., 2014). PCR and Southern blot analysis were conducted with genomic DNA from the transformants to confirm integration of the p188CpPKS1 vector into the fungal genome (Song et al., 2013).

\section{Analysis of the transformants}

Production of phleichrome from recombinant C. parasitica was analyzed using thin-layer chromatography (TLC) followed by liquid chromatography (LC/MS/MS). Briefly, EtOAc was used to extract phleichrome from mycelia on cellophane membranes overlaying agar plates. The crude extract was then resolved using TLC on silica gel with a resolving solution $\left(\mathrm{CH}_{2} \mathrm{Cl}_{2} / \mathrm{MeOH}\right.$ $=19: 1, \mathrm{v} / \mathrm{v}$ ) and purified phleichrome as a control ( $\mathrm{Yi}$ et al., 2011). The band with the same mobility as that of the purified phleichrome was scraped out from the silica gel and dissolved in methanol, and the presence of phleichrome was further analyzed by LC/MS/MS.

\section{LC/MS/MS analysis}

A Surveyor HPLC on line with an Agilent 6410B (Agilent Technology, Wilmington, DE, USA) LC/MS/MS mass spectrometer equipped with an ESI source was employed. Separations were performed on an analytical reverse phase column (Agilent XDB_C18, $5 \mu \mathrm{m}, 2.0 \mathrm{~mm} \times 15 \mathrm{~cm}$ ). The injection volume was 5 $\mu \mathrm{l}$. The mobile phases were as follows: $A=$ water, $0.1 \%$ formic acid, and $B=$ acetonitrile, $0.1 \%$ formic acid. Lipopeptides were eluted using a linear gradient of $10 \%$ B to $100 \%$ buffer for 20 $\mathrm{min}$ at a flow rate of $0.23 \mathrm{ml} / \mathrm{min}$. lons were generated in positive ionization mode using an electrospray ionization interface. Fragmenter potential was set to $130 \mathrm{~V}$, the interface heater was set to $300^{\circ} \mathrm{C}$, and the scan range was $\mathrm{m} / \mathrm{z} 100$ to 1000 . The metabolic profile of the TLC-purified pigment from the transformed C. parasitica was compared with the profile for purified phleichrome from $C$. phlei obtained in previous studies (Lee et al., 2007; Yi et al., 2011).

\section{RESULTS AND DISCUSSION}

Bioinformatic analyses of a gene involved in phleichrome biosynthesis

Structural comparison with other fungal perylenequinones revealed that phleichrome is a close analogue of cercosporin. The only gross structural differences between the two substances are the two methoxy groups in phleichrome instead of the methylenedioxy group of cercosporin (Arnone et al., 1985). Cercosporin is known to be synthesized via the polyketide metabolic pathway using acetate and malonate subunits (Choquer et al., 2005; Kurobane et al., 1981; Kusari et al., 2009; Liao and

Table 1. Primers for semi-quantitative $P C R$ analysis

\begin{tabular}{llll}
\hline Target gene & Forward sequence $\left(5^{\prime}-3^{\prime}\right)$ & Reverse sequence $\left(5^{\prime}-3^{\prime}\right)$ & Product size $(\mathrm{bp})$ \\
\hline Cppks1 & ATGGGTCGTCTTGCCCTTGTGAC & AGCTGATCTCGTGAGCATCG & 646 \\
Cppks3 & ACATCAAACCACACGAGGC & TCCTGATTGACGCCTGTCT & 681 \\
$\beta$-tub & ACTCTCTCGGTGGTGGTA & CTTGGGGTCGAACATCTG & 482 \\
\hline
\end{tabular}



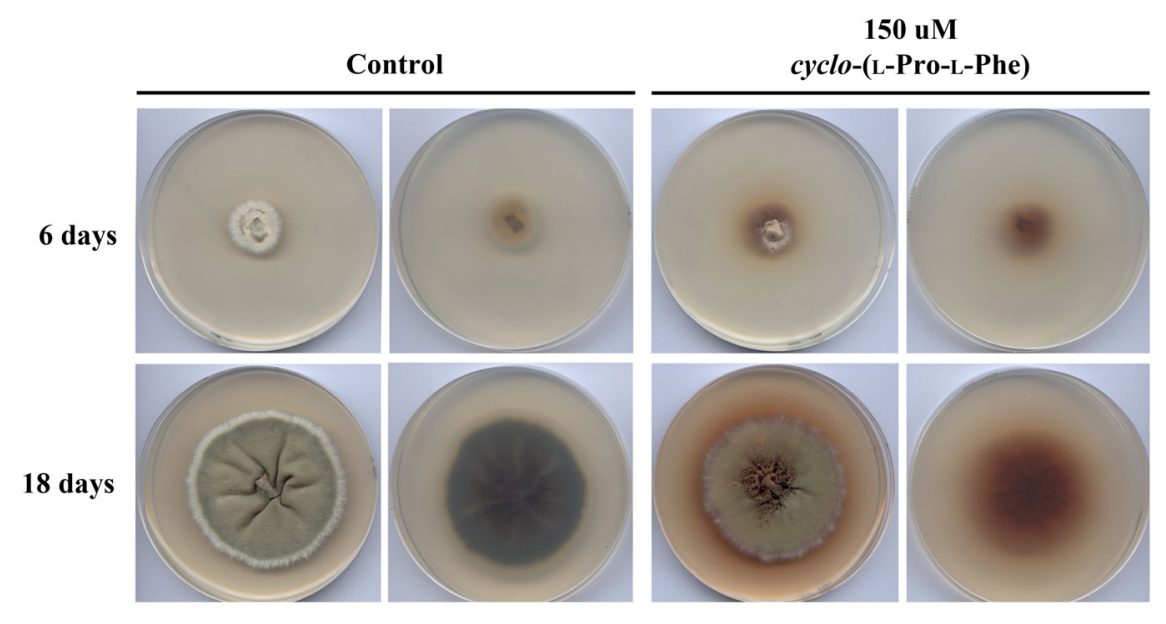

Fig. 1. Phleichrome production from the mycelia of wild-type C. phlei grown on PDA supplemented with $150 \mu \mathrm{M}$ cyclo-(L-Pro-L-Phe). Phleichrome production without induction as a control is also shown. Left panels demonstrate the characteristics of each colony from above, while right panels display the characteristics from the bottom of the plate.
Chung, 2008; Okubo et al., 1975). Structural similarity suggests that phleichrome is also synthesized via the polyketide pathway, which in fungi is orchestrated by polyketide synthases (PKS), which are multimeric enzymes that function analogously to fatty acid synthases joining carboxylic acid units in a stepwise fashion (Crawford and Townsend, 2010). All PKSs are currently divided into three general classes according to the organization of their active sites; fungal PKSs are multidomain systems (iterative type I) (Crawford and Townsend, 2010; Hutchinson and Fujii, 1995). In our previous study, we cloned four C. phlei PKS genes, which included three representative subclasses of PKS based on the level of reductive processing during chain assembly (So et al., 2012). In general, fungal aromatic polyketides, including phleichrome, are known to be synthesized by nonreducing (NR)-PKSs (Crawford and Townsend, 2010). Phylogenetic analysis of the four cloned $C$. phlei PKS genes indicated that the Cppks1 protein product appeared to be a NR-PKS (So et al., 2012). It was most closely related to other fungal PKS genes involved in the synthesis of red and orange perylenequinone pigments, including close analogues of phleichrome such as cercosporin (Choquer et al., 2005) and elsinochromes (Liao and Chung, 2008). In addition, encoded proteins of Cppks1 and two other genes for cercosporin and elsinochrome biosynthesis consisted of five catalytic domains, including keto synthase (KS), acyltransferase (AT), thioesterase/claisen cyclase (TE/CYC), and two consecutive acyl carrier proteins (ACP), that were distributed at comparable distances in the same order and at a similar length (Choquer et al., 2005; Liao and Chung, 2008; So et al., 2012). Altogether, our results suggested that Cppks1 is responsible for the biosynthesis of phleichrome via the fungal polyketide pathway.

\section{Regulation of the expression of Cppks1}

In our previous studies, we found that phleichrome production was significantly increased by addition of $150 \mu \mathrm{M}$ cyclo-(L-Pro- $\mathrm{L}^{-}$ Phe) synthetic inducer into the culture media (Fig. 1). Thus, we analyzed levels of the Cppks1 transcript in response to the inducer using semi-quantitative RT-PCR. Total RNA was extracted from cultures at 6 and 18 days postinoculation. These timepoints were chosen because discernable pigmentation was observed on the PDA plates by 6 days postinoculation and the maximum amount of phleichrome was produced by 18 days postinoculation. As shown in Fig. 2, supplementation of the

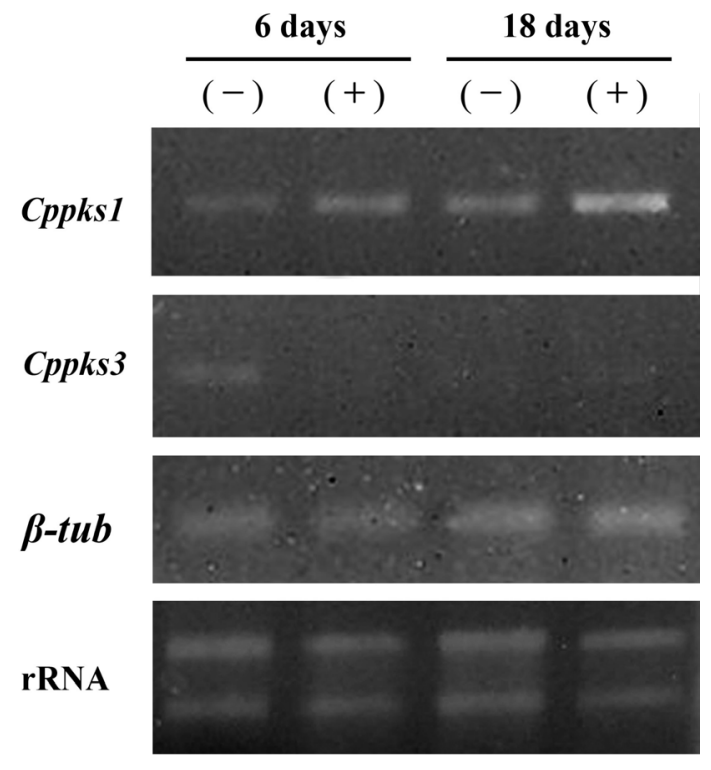

Fig. 2. Semi-quantitative RT-PCR analysis of Cppks1 transcript levels relative to levels of $\beta$-tubulin $(\beta$-tub). Total RNA was extracted 6- and 18-days after induction. Accumulation of Cppks1 transcript was compared with and without an induction. Experimental results were normalized to $\beta$-tub gene and the PKS gene Cppks3, a member of the HR-PKS subclass, was analyzed as an internal control. Note that equal amounts of RNA samples were loaded as shown in the bottom panel by the expression level of $\beta$-tub gene showing similar band intensities among samples and a representative ethidium bromide-stained rRNA bands from one of three independent experiments.

synthetic inducer into culture significantly increased the accumulation of Cppks1 transcript. The level of Cppks1 transcript increased along with incubation time, similar to the temporal pattern of phleichrome production under induction conditions. However, the expression of Cppks3, which is an HR-PKS, did not change in response to the synthetic inducer supplementation. The coordinated response of Cppks1 transcript to the inducer suggests that Cppks1 gene is associated with phleichrome biosynthesis. 
WT

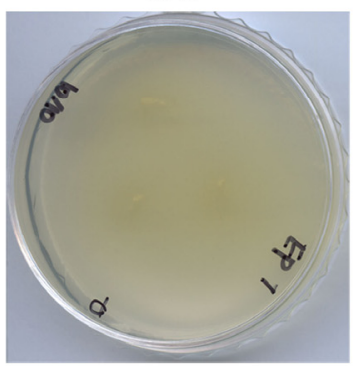

TNF \#44

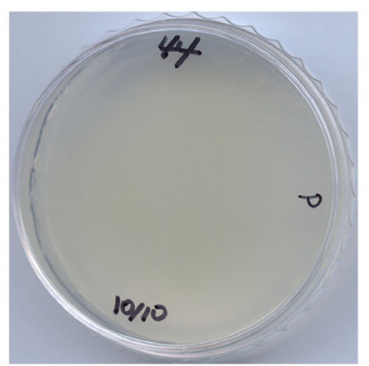

TNF \#16

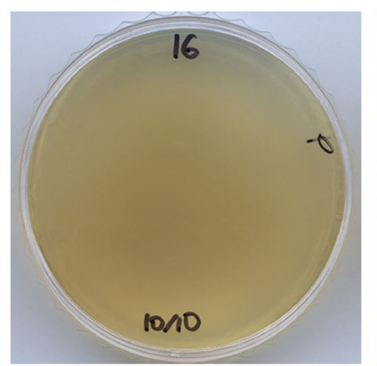

TNF \#56

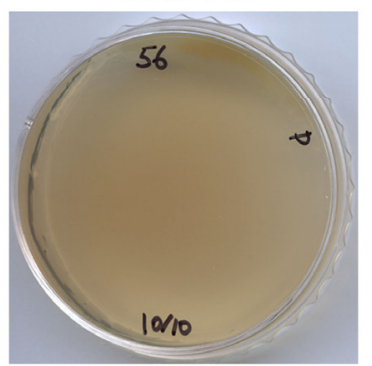

Fig. 3. Pigmentation of Cppks1-transformed C. parasitica. The bottoms of plates containing colonies after 10 days of culture on PDA supplemented with $100 \mathrm{mg} / \mathrm{L}$ of L-methionine and $1 \mathrm{mg} / \mathrm{L}$ of biotin (PDAmb) are shown. Strains used, which are indicated above the panel, were wild-type C. parasitica EP155/2 strain and three single-spored transformants (TNF\#44, \#16, and \#56). Note that TNF\#16 and \#56 had more pinkish pigments at the bottom compared to wild type.

\section{Analysis of C. parasitica transformants}

Heterologous expression is one of the most efficient methods for functional analysis of a given cloned gene. C. parasitica is a genetically tractable organism (Churchill et al., 1990) capable of producing highly complexed pigments such as skyrin, oxyskyrin, and rugulosin via the polyketide pathway (Shibata, 1973). Therefore, we expressed Cppks1 in C. parasitica to see if the resulting recombinant fungus would produce phleichrome.

Thirty putative transformants were selected from the top agar plates supplemented with $150 \mu \mathrm{g} / \mathrm{ml}$ geneticin, passaged three times on selective media supplemented with $50 \mu \mathrm{g} / \mathrm{ml}$ geneticin, and single-spore isolated prior to further analyses. Mitotic stability of each transformant was confirmed by successive transfers alternating on selective and non-selective media. In order to detect integration of the transforming vector into the chromosome of C. parasitica, PCR analysis using Cppks1-specific primers was conducted. All 30 transformants were positive for the 646-bp PCR amplicon, indicating stable integrative transformants.

Because phleichrome is responsible for the characteristic deep red pigmentation in the mycelia and culture medium, we looked for changes in the colour of colonies in single-spored transformants. As shown in Fig. 3, two colonies became pinkish over time, in contrast to the original orange colour, as culture aged. However, no discernible changes in any of the other characteristics, including growth rate and sporulation, were observed in these transformants. Therefore, we assessed the presence of phleichrome in these selected transformants. TLC analysis using an EtOAc extract of the mycelia revealed the presence of yellowish pigment at an Rf value of 0.24 , the same $\mathrm{Rf}$ as for purified phleichrome (Fig. 4A). However, these pigments were also present in wild-type $C$. parasitica. These results indicated that TLC was not sensitive enough to differentiate phleichrome from other residual pigments, such as skyrin and oxyskyrin. The amount of heterologous phleichrome produced may also have been too low for detection by TLC.

Thus, pigment spots migrating at the same Rf value as purified phleichrome on TLC plates were scraped off and extracted with methanol, and the presence of phleichrome was determined by LC/MS/MS analysis with purified phleichrome from $C$. phlei as a control. As shown in Fig. 4B, methanol extract of TLC spots from recombinant $C$. parasitica had a new peak with the same retention time as the control phleichrome, and the molecular weight of the corresponding peak was the same as that of phleichrome, confirming the presence of phleichrome in ex- tracts. These results clearly indicated that phleichrome is synthesized via the polyketide pathway and that Cppks1 encodes a protein responsible for the biosynthesis of phleichrome. It would be of great interest to carry out functional analysis of the Cppks1 gene product using gene replacement or silencing. In addition to the PKS gene, tailoring proteins that catalyze subsequent reactions including oxidation, hydration, methylation, and hydroxylation are also required for successful cercosporin biosynthesis (Chen and Nuss, 2007). C. parasitica does not produce phleichrome but does produce secondary metabolites such as skyrin, oxyskyrin, and rugulosin via the polyketide pathway (Shibata, 1973).

Genome survey of C. parasitica suggested the presence of at least $31 \mathrm{PKS}$ genes and/or clusters (http://genome.jgipsf.org/Crypa1/Crypa1.home.html). Thus, genes involved in the processing of the core component of $C$. parasitica may be involved in further processing of phleichrome. In addition, heterologous production of a fungal polyketide in the yeast Saccharomyces cerevisiae requires co-expression of the corresponding PKS gene and a heterologous 4'-phosphopantetheinyl transferase (PPTase) (Kealey et al., 1998; Wattanachaisaereekul et al., 2007). PPTases catalyze the post-translational modification of proteins by the covalent attachment of a 4'phosphopantetheine moiety of coenzyme $A$ to a conserved serine residue of an inactive form of $A C P$, resulting in conversion to the active form during the biosynthesis of metabolites (Walsh et al., 1997). Interestingly, no PPTase domain or genes were found in the gene cluster for cercosporin biosynthesis. Therefore, the successful heterologous production of phleichrome suggests that there was an appropriate PPTase that acted in trans in C. parasitica, that converted heterologously expressed inactive apo-CpPKS1 to active holoCpPKS1 by modifying the ACP domain of CpPKS1 with a 4'phosphopantetheine moiety. Almost all organisms that utilize more than one 4'-phosphopantetheine-dependent pathway also have more than one PPTase (Gehring et al., 1998; Quadri et al., 1998). A genome survey of $C$. parasitica suggested the presence of three PPTase genes (http://genome.jgi-psf.org/Crypa1/ Crypa1.home.html). However, filamentous fungi such as $\mathrm{Neu}$ rospora crassa, $A$. fumigatus, $A$. nidulans, and Fusarium fujikuroi harbor only a single PPTase (Kim et al., 2015).

Genes involved in the secondary metabolite pathways in filamentous fungi are often organized in clusters (Keller et al., 2005). Therefore, we are currently characterizing the loci surrounding Cppks1 to determine if genes involved in the biosyn- 
$\boldsymbol{A}$

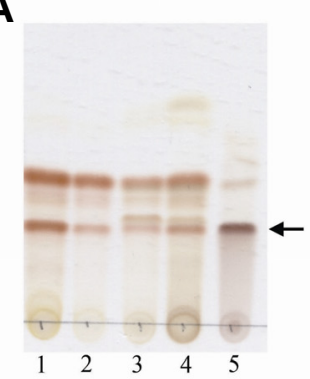

B

(i)

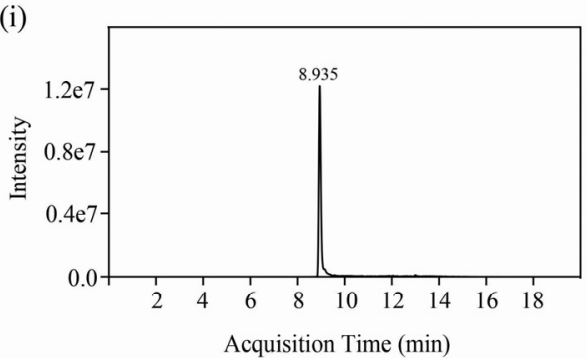

(ii)

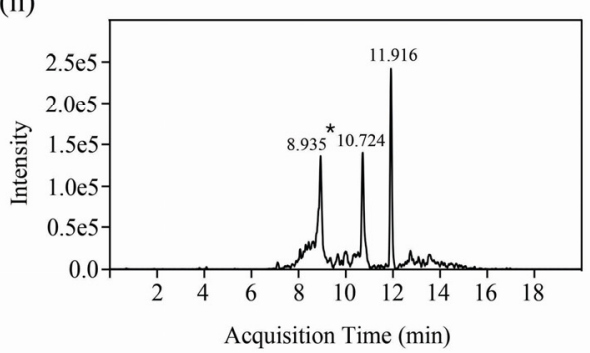

(iii)

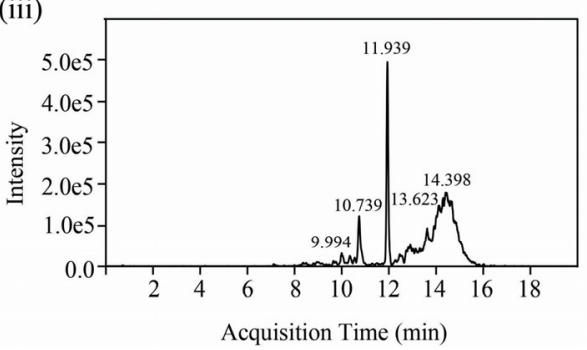

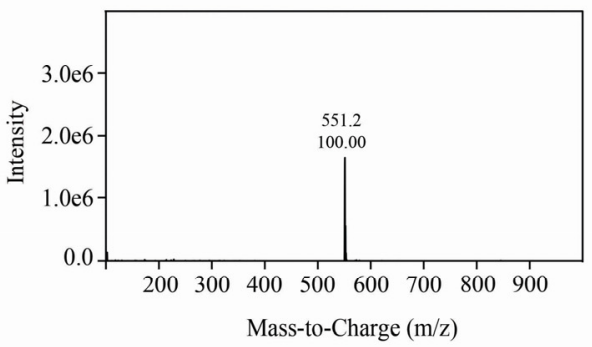

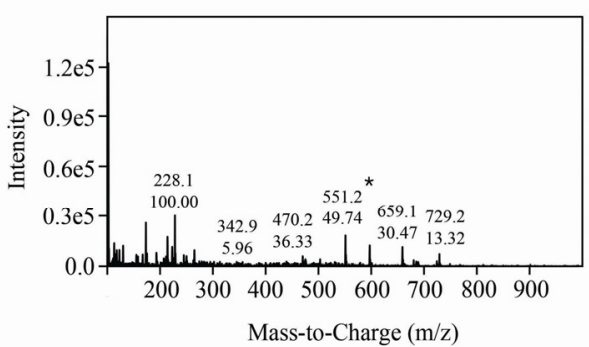

Fig. 4. LC-MS analysis of phleichrome in recombinant $C$. parasitica. (A) TLC analysis of the ethyl acetate extracted pigment is shown. Lanes 1-4 show sample preparations from wild-type C. parasitica and three representative transformants (TNF\#44, \#16, and \#56). Lane 5 shows purified phleichrome from previous studies (Lee et al., 2007) as a control. The arrow indicates the expected spot for phleichrome. (B) LCMS analysis of the methanol extract of the corresponding spots on the TLC plate. LC-MS profile of the extract from the representative recombinant $C$. parasitica (TNF\#16) expressing the Cppks1 gene (ii) was compared to the profile of the extract from wild-type C. parasitica (iii) and the phleichrome standard (i). Note that the new peak corresponding to the retention time of purified phleichrome is marked by an asterisk. Mass analysis of the corresponding peak $\left(^{*}\right)$ from the recombinant C. parasitica matched that of the purified phleichrome. thesis of phleichrome are clustered. Additionally, Cppks1 may not be the only gene for phleichrome synthesis. The survey of genomic library suggested that more NR-PKS genes remain to be identified in addition to the four PKS genes cloned in a previous study (So et al., 2012). Further studies are required to characterize all cloned PKS genes. Functional analysis of Cppks1 gene will allow determination of the redundancy of the phleichrome gene.

\section{ACKNOWLEDGMENTS}

This work was supported by the Bio-industry Technology Development Program, Ministry for Food, Agriculture, Forestry and Fisheries, Republic of Korea, and in part by the NRF grants by MSIP (No. 2008-0061897 and 2013R1A1A2012433). This research was also supported in part by the Korea Research Council of Fundamental Science \& Technology (Joint Degree and Research Center for Biorefinery) and in part by "Cooperative Research Program for Agricultural Science \& Technology Development (Project No. PJ009998012015)" Rural Development Administration, Republic of Korea. We thank the Institute of Molecular Biology and Genetics at Chonbuk National University for kindly providing the facilities for this research. This paper was supported by research funds of Chonbuk National University in 2015. K. K. So was supported by BK21 PLUS program in the Department of Bioactive Material Sciences.

\section{REFERENCES}

Arnone, A., Camarda, L., Nasini, G., and Merlini, L. (1985). Secondary mould metabolites. Part 13. Fungal Perylenequinones: phleichrome, isophleichrome, and their endoperoxides. J. Chem. Soc. Perkin Trans. I 1387-1392.

Baek, J.H., Park, J.A., Kim, J.M., Oh, J.M., Park, S.M., and Kim, 
D.H. (2014). Functional analysis of a tannic-acid-inducible and hypoviral-regulated small heat-shock protein Hsp24 from the chestnut blight fungus Cryphonectria parasitica. Mol. Plant Microbe Interact. 27, 56-65.

Chen, R.E., and Thorner, J. (2007). Function and regulation in MAPK signaling pathways:lessons learned from the yeast Saccharomyces cerevisiae. Biochim. Biophys. Acta. 1773, 13111340.

Choi, G.H., and Nuss, D.L. (1990). Nucleotide sequence of the glyceraldehyde-3-phosphate dehydrogenase gene from Cryphonectria parasitica. Nucleic Acids Res. 18, 5566.

Choquer, M., Dekkers, K.L., Chen, H.Q., Cao, L., Ueng, P.P., Daub, M.E., and Chung. K.R. (2005). The CTB1 gene encoding a fungal polyketide synthase is required for cercosporin biosynthesis and fungal virulence of Cercospora nicotianae. Mol. Plant Microbe Interact. 18, 468-476.

Churchill, A.C.L., Ciufetti, L.M., Hansen, D.R., Van Etten, H.D., and Van Alfen, N.K. (1990). Transformation of the fungal pathogen Cryphonectria parasitica with a variety of heterologous plasmids. Curr. Genet. 17, 25-31

Crawford, J.M., and Townsend, C.A. (2010). New insights into the formation of fungal aromatic polyketides. Nat. Rev. Microbiol. 8, 879-889.

Daub, M.E., and Ehrenshaft, M. (2000). The photoactivated cercospora toxin cercosporin: Contributions to plant disease and fundamental biology. Annu. Rev. Phytopathol. 38, 461-490.

Daub, M.E., Herrero, S., and Chung, K.R. (2005). Photoactivated perylenequinone toxins in fungal pathogenesis of plants. FEMS Microbiol. Lett. 252, 197-206.

Du, M., and Maunder, D. (2010). Method for preparing hypocrellin. USA Patent 7816563 B1.

Gehring, A.M., Mori, I., and Walsh, C.T. (1998). The nonribosomal peptide synthetase HMWP2 forms a thiazoline ring during biogenesis of yersiniabactin, an iron-chelating virulence factor of Yersinia pestis. Biochemistry 37, 2648-2659.

Hudson, J.B., and Towers, G.H. (1991). Therapeutic potential of plant photosensitizers. Pharmacol. Ther. 49, 181-222

Hutchinson, C.R., and Fujii, I. (1995). Polyketide synthase gene manipulation - a structure -function approach in engineering novel antibiotics. Annu. Rev. Microbiol. 49, 201-238.

Kealey, J.T., Liu, L., Santi, D.V., Betlach, M.C., and Barr, P.J. (1998). Production of a polyketide natural product in nonpolyketide-producing prokaryotic and eukaryotic hosts. Proc. Natl. Acad. Sci. USA 95, 505-509.

Keller, N.P., Turner, G., and Bennett, J.W. (2005). Fungal secondary metabolism - from biochemistry to genomics. Nat. Rev. Microbiol. 3, 937-947.

Kim, D.H., and Martyn, R,D. (1995). Transformation of Fusarium oxysporum f. sp. niveum to hygromycin B resistance and altered pathogenicity. Mol. Cells 5, 658-667

Kim, J.A., Kim, J.M., Kim, H.G., Kim, B.T., Hwang, K.J., Park, S.M., Yang, M.S., and Kim, D.H. (2009). Protoplast-mediated transformation of the filamentous fungus Cladosporium phlei: Evidence of tandem repeats of the integrative transforming vector. Plant Pathol. J. 25, 179-183.

Kim, J.M., Song, H.Y., Choi, H.J., So, K.K., Kim, D.H., Chae, K.S., Han, D.M., and Jahng, K.Y. (2015). Characterization of NpgA, a 4-phosphopantetheinyl transferase of Aspergillus nidulans, and evidence of its involvement in fungal growth and formation of conidia and cleistothecia for development. J. Microbiol. 15, 21-32.

Kurobane, I., Vining, L.C., Mclnnes, A.G., Smith, D.G., and Walter, J.A. (1981). Biosynthesis of elsinochromes C and D. Pattern of acetate incorporation determined by $13 \mathrm{C}$ and $2 \mathrm{H} \mathrm{nmr1}$. Can. J. Chem. 59, 422-430.

Kusari, S., Zuhlke, S., Borsch, T., and Spiteller, M. (2009). Positive correlations between hypericin and putative precursors detected in the quantitative secondary metabolite spectrum of Hypericum. Phytochemistry 70, 1222-1232.

Kwon, B.R., Kim, M.J., Park, J.A., Chung, H.J., Kim, J.M., Park, S.M., Yun, S.H., Yang, M.S., and Kim, D.H. (2009). Assessment of the core cryparin promoter from Cryphonectria parasitica for heterologous expression in filamentous fungi. Appl. Microbiol. Biotechnol. 83, 339-348.

Lee, J.K., Kim, B.T., Kim, J.A., Chung, H.J., Park, S.M., Yang M.S., Hwang, K.J., and Kim, D.H. (2007). Cultural characteristics and extraction of the fungal pigment phleichrome from the phytopathogenic fungus Cladosporium phlei. Biotechnol. Bioprocess Eng. 12, 508-515.

Liao, H.L., and Chung, K.R. (2008). Genetic dissection defines the roles of elsinochrome phytotoxin for fungal pathogenesis and conidiation of the citrus pathogen Elsinoe fawcettii. Mol. Plant Microbe Interact. 21, 469-479.

Mullaney, E.J., Hamer, J.E., Roberti, K.A., Yelton, M.M., and Timberlake, W.E. (1985). Primary structure of the trpC gene from Aspergillus nidulans. Mol. Gen. Genet. 199, 37-45.

Nguyen, Q.B., Kadotani, N., Kasahara, S., Tosa, Y., Mayama, S., and Nakayashiki, H. (2008). Systematic functional analysis of calcium-signalling proteins in the genome of the rice-blast fungus, Magnaporthe oryzae, using a high-throughput RNA-silencing system. Mol. Microbiol. 68, 1348-1365.

Okubo, A., Yamazaki, S., and Fuwa, K. (1975). Biosynthesis of cercosporin. Agric. Biol. Chem. 39, 1173-1175.

Olivo, M., and Chin, W. (2006). Perylenequinones in photodynamic therapy: cellular versus vascular response. J. Environ. Pathol Toxicol. Oncol. 25, 223-237.

Park, S.M., Choi, E.S., Kim, M.J., Cha, B.J., Yang, M.S., and Kim, D.H. (2004). Characterization of HOG1 homologue, CpMK1, from Cryphonectria parasitica and evidence for hypovirusmediated perturbation of its phosphorylation in response to hypertonic stress. Mol. Microbiol. 51, 1267-1277.

Park, J.A., Kim, J.M., Park, S.M., and Kim, D.H. (2012) Characterization of CpSte11, a MAPKKK gene of Cryphonectria parasitica, and initial evidence of its involvement in the pheromone response pathway. Mol. Plant Pathol. 13, 240-250.

Quadri, L.E., Sello, J., Keating, T.A., Weinreb, P.H., and Walsh, C.T. (1998). Identification of a Mycobacterium tuberculosis gene cluster encoding the biosynthetic enzymes for assembly of the virulence-conferring siderophore mycobactin. Chem. Biol. 5, 631645.

Shibata, S. (1973). Some recent studies on the metabolites of fungi and lichens. Pure Appl. Chem. 33, 109-128.

So, K.K., Kim, J.M., Nguyen, N.L., Park, J.A., Kim, B.T., Park, S.M. Hwang, K.J., and Kim, D.H. (2012). Rapid screening of an ordered fosmid library to clone multiple polyketide synthase genes of the phytopathogenic fungus Cladosporium phlei, J. Microbiol. Methods 9, 412-419.

So, K.K., Jo, I.S., Chae, M.S., Kim, J.M., Chung, H.J., Yang, M.S. Kim, B.T., Kim, J.K., Choi, J.K., and Kim, D.H. (2015). Improved production of phleichrome from the phytopathogenic fungus Cladosporium phlei using synthetic inducers and photodynamic ROS production by phleichrome. J. Biosci. Bioeng. 119, 289-296.

Song, M.Y., Kim, C.Y., Han, M., Ryu, H.S., Lee, S.K., Sun, L., He, Z., Seo, Y.S., Canal, P., Ronald, P.C., and Jeon, J.S. (2013). Differential requirement of Oryza sativa $R A R 1$ in immune receptor-mediated resistanve of rice to Magnaporthe oryzae. Mol Cells 35, 327-334.

Walsh, C.T., Gehring, A.M., Weinreb, P.H., Quadri, L.E., and Flugel R.S. (1997). Post-translational modification of polyketide and nonribosomal peptide synthases. Curr. Opin. Chem. Biol. 1, 309315.

Wattanachaisaereekul, S., Lantz, A.E., Nielsen, M.L., Andresson, O.S., and Nielsen, J. (2007). Optimization of heterologous production of the polyketide 6-MSA in Saccharomyces cerevisiae. Biotechnol. Bioeng. 97, 893-900.

Yi, M.H., Kim, J.A., Kim, J.M., Park, J.A., Kim, B.T., Park, S.M., Yang, M.S., Hwang, K.J., and Kim, D.H. (2011). Characterization of a mutant strain of a filamentous fungus Cladosporium phlei for the mass production of the secondary metabolite phleichrome. J. Microbiol.49, 680-683.

Yoshihara, T., Shimanuki, T., Araki, T., and Sakamura, S. (1975) Phleichrome, a new phytotoxic compound produced by Cladosporium phlei. Agric. Biol. Chem. 39, 1683-1684. 